\title{
Dispersal-Mediated Coexistence of Indirect Competitors in Source-Sink Metacommunities
}

\author{
Toshiyuki NAmBA \\ Department of Biological Science, Graduate School of Science \\ Osaka Prefecture University \\ Daisen-cho 2-1, Sakai-ku, Sakai, 590-0035, Japan \\ Tel: +81-(0)72-222-4811 (ext. 4344) \\ Fax: +81-(0)72-222-4791 \\ E-mail: tnamba@b.s.osakafu-u.ac.jp
}

Received July 2, 2006

Revised September 23, 2006

\begin{abstract}
The source-sink dynamics is a major hypothesis to explain dispersal-mediated coexistence of locally exclusive competitors. We study Lotka-Volterra diffusive models of indirect competition in patchy metacommunities. In a model of exploitative competition, we numerically show that the effect of resource movement on the coexistence depends on demographic factors that create source-sink structures and that the dispersal rate of the superior competitor need not be higher than that of the inferior to promote dispersal-mediated coexistence. In a model of apparent competition, we analytically prove that dispersal can make coexistence possible even if any patches are sinks for the inferior resource species. The requirement for this coexistence is the lower dispersal rate of the inferior competitor. We conclude that dispersal among patches can be a mechanism to save inferior indirect competitors from regional extinction and that the level of spatial heterogeneity need not be so high to reverse the competitive rankings among patches.
\end{abstract}

Key words: exploitative competition, apparent competition, metacommunity, source-sink dynamics, dispersal-mediated coexistence

\section{Introduction}

A "metacommunity" is defined as a set of local communities coupled by dispersal or movement of one or more component species in a heterogeneous landscape (Hanski and Gilpin [16], Wilson [70]). Metacommunity dynamics are influenced by both the species interactions and dispersal among patches (Leibold et al. [37], Holyoak et al. [26, 27]).

The most striking patterns and processes in metacommunities are the facilitation of species coexistence and the maintenance of biodiversity (Amarasekare [3], Chase et al. [11], Hoopes et al. [28], Mouquet et al. [43]). Numerous models and mechanisms have been proposed to explain spatial coexistence of locally exclusive competitors (Hanski and Gilpin [17], Tilman and Kareiva [67], Dieckmann et al. [15]). Now, it is necessary to synthesize them (Chesson [13, 14], Amarasekare [3], Chase et al. [11], Hoopes et al. [28], Mouquet et al. [43]) and investigate the effect of simultaneous operations of multiple spatial mechanisms (Amarasekare et al. [4]).

Leibold et al. [37] and Holyoak et al. [26, 27] recently classified metacommunity models into four conceptual perspectives, (1) "patch dynamic," (2) "species sorting," (3) "mass effect," and (4) "neutral" perspectives, to illuminate different aspects of spatial community dynamics. In two of the four perspectives, the patch 
dynamics and mass effect, dispersal plays important roles to promote spatial coexistence of locally exclusive competitors.

The patch dynamic perspective is a direct descendant of the classical patch occupancy models (Levins $[40,41]$ ) that assume identical patches and spatial dynamics dominated by local extinction and recolonization. The competition-colonization trade-off (reviewed in Kneitel and Chase [34]) is a popular hypothesis that explains spatial coexistence of locally exclusive competitors in this framework (Tilman [66], Tilman et al. [68], Yu and Wilson [71], Calcagno et al. [9]). It suggests that locally inferior competitors can escape regional extinction if they are good colonizers and exploit gaps in the landscape left unused by the superior. This implies that inferior competitors should disperse at faster rates or farther away to become good colonizers when they are not fecund enough (Levins and Culver [42], Horn and MacArthur [29], Hastings [18], Tilman [66], Tilman et al. [68]). However, short range dispersal of inferior competitors can sometimes facilitate coexistence, by allowing them to quickly fill and exploit empty patches (Bolker and Pacala [7]), concentrating lowerdensity species in areas where the growth rates are higher (Chesson [14], Snyder and Chesson [61]) or promoting spatial segregation of competitors that have different interaction neighborhoods among herospecific and conspecific individuals (Murrell and Law [49]). It should also be noted that this mechanism owes the coexistence of a potentially infinite number of species to the assumptions of the strict competitive hierarchy (Pacala and Rees [54], Bolker and Pacala [7], Adler and Mosquera [2]) and the instantaneous displacement of the inferior by the dominant (Yu and Wilson [71], Calcagno et al. [9]).

The mass effect perspective focuses on immigration and emigration. In spatially heterogeneous habitats, locally inferior species in unfavorable patches may be rescued by immigrants from communities where they are good competitors (Brown and Kodric-Brown [8], Shmida and Whittaker [60]). This perspective assumes that habitats are spatially heterogeneous in the sense that competitive rankings vary within the spatial extent of the landscape (Mouquet and Loreau [44, 45], Amarasekare [3], Amarasekare et al. [4], Mouquet et al. [43]). Thus, it is almost equivalent to or an important limiting case (Holt [22]) of the source-sink dynamics (Namba [50], Holt [21], Pulliam [56], Pulliam and Danielson [57]) and closely related to the spatial storage effect (Chesson $[12,13,14]$ ). The source-sink dynamics are usually described by either one of the hierarchical patch occupancy models (or weighted competitive lottery models; Chesson [12], Iwasa and Roughgarden [32], Muko and Iwasa [47, 48], Mouquet and Loreau [44, 45], Amarasekare et al. [4]) or the explicit within-patch dynamics models describing species abundances and interactions within patches (Levin [39], Kishimoto [33], Namba et al. [52], Amarasekare and Nisbet [5]).

Levin [39] considered a Lotka-Volterra model of competition in two identical patches. He assumed symmetric local dynamics and allowed each species to dominate numerically in patches it occupies prior to the other species. Then, dispersal from patches with abundant populations can sustain impoverished populations in patches occupied beforehand by competitors unless the rate of dispersal is too high 
to homogeneize the habitat. Amarasekare and Nisbet [5] explored conditions for coexistence when competitive rankings are spatially variable and Kishimoto [33] found conditions on dispersal rates for coexistence of infinitely many competitors.

However, coexistence in the Lotka-Volterra metapopulation models requires neither the priority effect nor the spatial heterogeneity in competitive rankings. Two competitors can coexist regionally even if one of them is inferior in any spatial region or in any patch throughout the habitat, in spatially continuous (Pacala and Roughgarden [55], Cantrell and Costner [10]) and patchy (Takeuchi [63]) competition-diffusion models of the Lotka-Volterra type. Coexistence is favored by a relatively small dispersal rate of the inferior and a moderate or relatively large dispersal rate of the superior (Pacala and Roughgarden [55], Takeuchi [63]).

In natural ecological communities, many species are linked through numerous trophic and competitive interactions. Thus, it is important to extend those findings to food web models of three or more species in spatially heterogeneous habitats. Recently, both the hierarchical patch occupancy models (Mouquet and Loreau [44, 45], Amarasekare et al. [4]) and explicit within-patch models (Holt [20, 21], Namba et al. [52]) of source-sink dynamics have been extended. In the latter, it has been found that dispersal can mediate coexistence of two consumers (predators) indirectly competing through a shared resource (prey) in a habitat in which the competitive rankings are spatially invariable (Namba and Hashimoto [51], Abrams and Wilson [1]). However, at least two important questions remain unresolved. First, in exploitative metacommunities, the effect of resource (prey) movement among patches has not yet been studied. On the other hand, it has been experimentally shown that dispersal of both predators and prey is important for persistence of protist predator-prey communities in subdivided habitats of connected bottles (Holyoak and Lawler [24, 25]). We can also find many examples of diffusion and range expansions of organisms in lower trophic levels (and thus are prey), such as plants, plankton, and insects (Shigesada and Kawasaki [59], Okubo and Levin [53]). Second, metacommunity models of another important indirect competition, apparent competition (Holt [19, 20], Namba et al. [52]), have not yet been fully studied, in particular, when resource species can move among patches and competitive rankings are spatially homogeneous.

In this article, we study two Lotka-Volterra models of diffusive indirect competitors in metacommunities of two different patches. First, in an exploitative competition model, we summarize the results of Namba and Hashimoto [51] and Abrams and Wilson [1]. Then, we numerically explore the effect of resource movement between the two patches and examine whether the resource movement promotes or demotes spatial coexistence of exploitative competitors and how the result depends on demographic factors (resource growth rate, carrying capacity or consumer mortality) which generate source-sink spatial structures in the metacommunities. We will also demonstrate that the higher dispersal rate of the superior competitor is not always needed for the dispersal-mediated coexistence, which was necessary in Namba and Hashimoto [51] and Abrams and Wilson [1] unless higher demographic rates of the inferior competitor save the latter. Second, in a metacommunity model of ap- 
parent competition, we will analytically show that dispersal-mediated coexistence can be realized even if the competitive rankings are spatially invariable, making use of bifurcation analysis and a Lyapunov function. The condition for coexistence will be that the inferior competitor is sedentary as in Namba and Hashimoto [51] and Abrams and Wilson [1]. We will also consider the case where the competitive rankings differ between the two patches and a true source-sink structure appears. Finally, we discuss ecological implications of our results and future directions.

\section{Exploitative competition}

A metacommunity model of exploitative competition which describes dynamics of two consumers (predators) sharing a common resource (prey) in a habitat of two different patches (Holt [21], Namba and Hashimoto [51], Abrams and Wilson [1]) can be written as follows;

$$
\begin{aligned}
& \frac{d R^{i}}{d t}=f\left(R^{i}\right) R^{i}-g_{1}\left(R^{i}\right) C_{1}^{i}-g_{2}\left(R^{i}\right) C_{2}^{i}-d_{r}\left(R^{i}-R^{j}\right), \\
& \frac{d C_{1}^{i}}{d t}=\left(-m_{1}{ }^{i}-p_{1} C_{1}^{i}+b_{1} g_{1}\left(R^{i}\right)\right) C_{1}^{i}-d_{1}\left(C_{1}^{i}-C_{1}^{j}\right), \\
& \frac{d C_{2}^{i}}{d t}=\left(-m_{2}{ }^{i}-p_{2} C_{2}{ }^{i}+b_{2} g_{2}\left(R^{i}\right)\right) C_{2}^{i}-d_{2}\left(C_{2}^{i}-C_{2}^{j}\right),
\end{aligned}
$$

where $(i, j)=(1,2)$ or $(2,1) . R^{i}, C_{1}{ }^{i}$, and $C_{2}{ }^{i}$ are respectively the densities of resource $R$ and consumers $C_{1}$ and $C_{2}$ in the $i$-th patch. $f\left(R^{i}\right)$ is the per capita rate of increase of resource in the $i$-th patch; $g_{k}\left(R^{i}\right)$ is the per capita rate of consumption, or the functional response of the $k$-th consumer, and $b_{k}$ is the conversion efficiency of the $k$-th consumer. $m_{k}{ }^{i}$ is the mortality of the $k$-th consumer in the $i$-th patch, $p_{k}$ is the coefficient of intraspecific competition or density-dependent mortality of the $k$-th consumer, and $d_{r}$ and $d_{k}(k=1,2)$ are the dispersal rates of resource and consumers respectively.

In non-spatial models of exploitative competition, two consumers exploiting a common resource compete indirectly through resource depletion. It is well-known that they can never coexist in equilibrium (Stewart and Levin [62], Koch [35]), unless some density dependence or interference competition is taken into consideration in the dynamics of consumer (predator) populations (Kostitzin [36]). There exists minimum resource density $R_{k}{ }^{*}$ necessary to sustain the consumer species $C_{k}$ in equilibrium (Tilman $[64,65]$ ). It is known as the Tilman's $R^{*}$ rule in resource competition theory (Tilman [64, 65]; originally developed by Leon and Tumpson [38]) that only the consumer species having minimum $R^{*}$ can survive in equilibrium since any other species declines at $R=\min _{k} R_{k}{ }^{*}$. However, when the functional responses are nonlinear and saturating, they can coexist (Koch [35], Hsu et al. [30, 31], Armstrong and McGehee [6]), since the competitive rankings vary temporally because of population oscillations if each species is an efficient resource exploiter respectively at lower or higher resource densities. 
Namba and Hashimoto [51] assumed the logistic resource growth, $f\left(R^{i}\right)=$ $r\left(1-R^{i} / K^{i}\right)$, and the Holling's type II functional responses, $g_{k}\left(R^{i}\right)=a_{k} R^{i} /(1+$ $\left.a_{k} h_{k} R^{i}\right)(k=1,2)$. They also assumed that only the resource carrying capacity $K^{i}$ is different between the two patches and ignored the resource dispersal rate $d_{r}$ presuming that the resource is a sessile organism. Starting from a case where each species has a favorable patch in which it excludes the other species in isolation, they numerically explored conditions for spatial coexistence, changing the carrying capacity in a patch. Consequently, they found that dispersal-mediate coexistence is possible, even if the competitive rankings of the consumers become identical in both patches. By the spatial difference in carrying capacities, the source-sink structure appears and emigration of the superior competitor from the relatively more productive patch releases the inferior from strong competitive pressure in the patch. Eventually, a lower rate of dispersal of the inferior enables it to persist in the entire habitat. Abrams and Wilson [1] analyzed a similar model of the LotkaVolterra type and found that coexistence is possible if an inferior consumer with the higher $R^{*}$ either has a lower movement rate or a more rapid demographic response to resource intake. They also verified that coexistence is possible in a wide range of conditions measured by $R^{*}$ and that an inferior competitor with a higher $R^{*}$ can exclude a superior exploiter with a lower $R^{*}$ from the entire habitat. Therefore, both in Namba and Hashimoto [51] and Abrams and Wilson [1], coexistence is facilitated when inferior exploiters (with higher $R^{*}$ ) are poorer dispersers if the competitive rankings are spatially homogeneous.

In the following, we numerically examine the effect of resource movement, which was ignored both in Namba and Hashimoto [51] and Abrams and Wilson [1]. We assume in equation (1) that $f\left(R^{i}\right)=r\left(1-R^{i} / K^{i}\right), g_{k}\left(R^{i}\right)=a_{k} R^{i}$. Here, we show two examples. In both examples, we assume that $a_{1}=a_{2}=1.0, b_{1}=1.0$, $b_{2}=0.5$, and $p_{1}=p_{2}=0.5$. In the first example (Fig. 1), the intrinsic rate of increase and carrying capacity of the resource are different between the two patches, $r_{1}=1.2, K_{1}=1.2, r_{2}=3.6, K_{2}=3.6$, and the mortalities of the consumers are the same between the two patches, $m_{1}{ }^{1}=m_{2}{ }^{1}=m_{1}{ }^{2}=m_{2}{ }^{2}=1.0$. In the second example (Fig. 2), the intrinsic rate of increase and carrying capacity of the resource are identical between the two patches, $r_{1}=r_{2}=3.6, K_{1}=K_{2}=3.6$, and the mortalities of the consumers differ between the two patches, $m_{1}{ }^{1}=m_{2}{ }^{1}=1.0$, $m_{1}^{2}=3.2, m_{2}^{2}=2.0$. In the absence of dispersal, the first consumer is superior and the second consumer goes to extinction in both patches. It should be noted that the following results do not depend on the assumed specific values of parameters, although dispersal mediated coexistence becomes more unlikely as the habitat tends to be more homogeneous. In the first example, both the resource and consumers become more abundant in the second patch, because the bottom-up effect of the higher intrinsic growth rate and carrying capacity enhances the resource and hence increases the consumer. However, in the second example, the consumer densities become lower and the resource density becomes higher in the second patch, because the top-down effect of the higher consumer mortalities reduces the consumers and hence increases the resource. This reverses the directions of flows of consumers and 
(a)

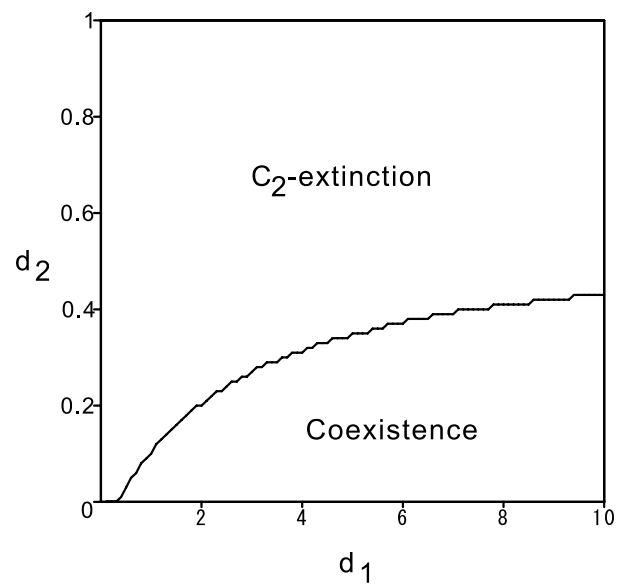

(b)

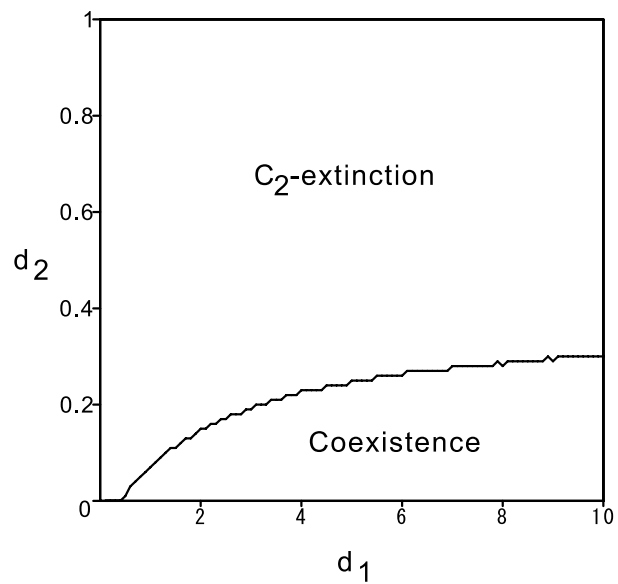

Fig. 1. Conditions for dispersal-mediated coexistence in a parameter space $d_{1}-d_{2}$. In a region denoted as "Coexistence," there is a stable positive steady state. In a region denoted as " $C^{2}$-extinction," a steady state in which $C^{2}$ is extinct is stable. Values of parameters are as follows; $p_{1}=p_{2}=0.5, b_{1}=1, b_{2}=0.5$, $m_{1}{ }^{1}=m_{2}{ }^{1}=m_{1}{ }^{2}=m_{2}{ }^{2}=1, r_{1}=1.2, K_{1}=1.2, r_{2}=3.6, K_{2}=3.6$, and $d_{r}=0$ in (a) and $d_{r}=0.1$ in (b).

(a)

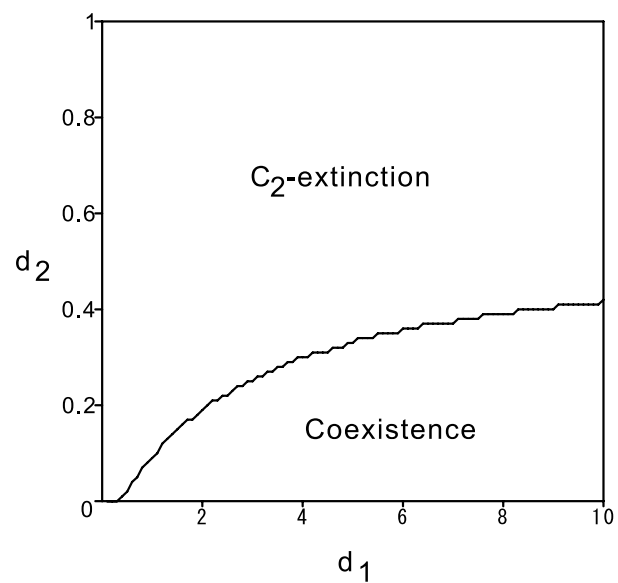

(b)

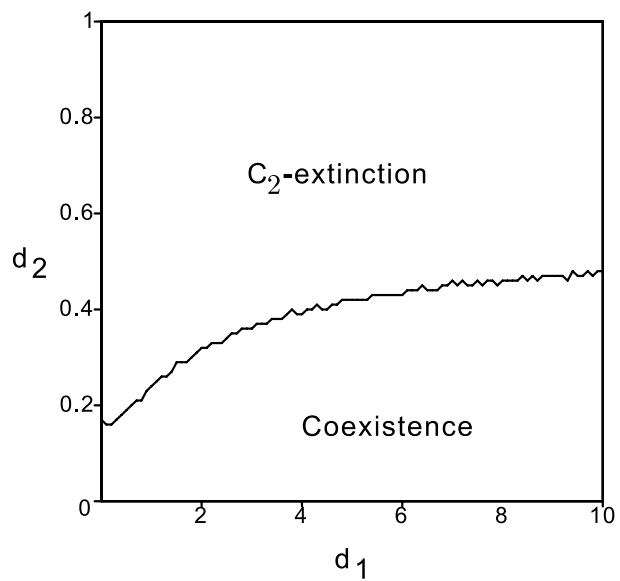

Fig. 2. Conditions for dispersal-mediated coexistence in a parameter space $d_{1}-d_{2}$. In a region denoted as "Coexistence," there is a stable positive steady state. In a region denoted as " $C^{2}$-extinction," a steady state in which $C^{2}$ is extinct is stable. Values of parameters are as follows; $p_{1}=p_{2}=0.5, b_{1}=1, b_{2}=0.5, m_{1}{ }^{1}=1$, $m_{2}{ }^{1}=1, m_{1}{ }^{2}=3.2, m_{2}{ }^{2}=2, r_{1}=3.6, K_{1}=3.6, r_{2}=3.6, K_{2}=3.6$, and $d_{r}=0$ in (a) and $d_{r}=10$ in (b). 
resource. The consumer population flows from the first patch to the second and the resource population flows from the second patch to the first.

In the first example, the resource dispersal reduces the difference in resource densities, thereby reducing the difference in consumer densities between the habitats and tend to lessen the source-sink spatial structure for the consumers. Therefore, higher dispersal rate of the resource makes coexistence of consumers more difficult (Fig. 1). At a fixed rate of resource movement, dispersal rate of the inferior consumer should be lower than that of the superior competitor for coexistence, for the outflow from a patch not to exceed the reproduction in that patch.

In the second example, the resource dispersal raises the resource level in the patch where consumer mortality is lower and resource density is low. Therefore, the resource dispersal enhances or subsidizes consumers in the recipient patch and promotes coexistence of the consumers (Fig. 2). Release of competitive pressure, or emigration of the superior competitor is not necessary for coexistence in this case. However, the superior may be extinct in the second patch if $d_{1}=0$, because the resource is washed out. Although the dispersal rate of the inferior competitor should not be too high not to be diluted out from the entire habitat, it need not be smaller than that of the superior.

Thus, resource dispersal can either promote or demote spatial coexistence of exploitative competitors, depending on which demographic factors contribute to create the source-sink spatial structures of the consumers. Requirements on dispersal rates of consumers for coexistence change also with the mechanisms to create the source-sink spatial structures.

\section{Apparent competition}

In this section, we consider a Lotka-Volterra metacommunity model of two resources (prey) sharing a common consumer (predator) in a habitat of two different patches (Holt [20], Namba et al. [52]);

$$
\begin{aligned}
& \frac{d R_{1}^{i}}{d t}=\left(r_{1}^{i}-a_{1}^{i} R_{1}^{i}-c_{1} C^{i}\right) R_{1}^{i}-d_{1}\left(R_{1}^{i}-R_{1}^{j}\right), \\
& \frac{d R_{2}{ }^{i}}{d t}=\left(r_{2}{ }^{i}-a_{2}{ }^{i} R_{2}{ }^{i}-c_{2} C^{i}\right) R_{2}{ }^{i}-d_{2}\left(R_{2}{ }^{i}-R_{2}{ }^{j}\right), \\
& \frac{d C^{i}}{d t}=\left(-m^{i}+b_{1} c_{1} R_{1}{ }^{i}+b_{2} c_{2} R_{2}{ }^{i}\right) C^{i}-d_{c}\left(C^{i}-C^{j}\right),
\end{aligned}
$$

where $(i, j)=(1,2)$ or $(2,1) . R_{1}{ }^{i}, R_{2}{ }^{i}$, and $C^{i}$ are respectively the densities of resources $R_{1}$ and $R_{2}$ and consumer $C$ in the $i$-th patch. $r_{k}{ }^{i}$ and $a_{k}{ }^{i}$ are respectively the intrinsic growth rate and the intraspecific competition coefficient of the $k$-th resource in the $i$-th patch; $c_{k}$ and $b_{k}$ are respectively the encounter rate of consumer with the $k$-th resource and the conversion efficiency; $m^{i}$ is the mortality of consumer in the $i$-th patch; $d_{1}, d_{2}$, and $d_{c}$ are respectively the dispersal rates of resources 1 and 2 and consumer. We assume, for simplicity, that only the intrinsic growth rates, intraspecific competition coefficients of resources, and consumer mortality can 
differ in two patches. In this notation, the superscripts $i$ and subscripts $k=1,2$ respectively denote the identities of patches and resource species.

In this model, alternate resources (prey) increase equilibrium abundance of consumer and reduce each other's equilibrium abundances. This indirect interaction among resources that share a common consumer was termed apparent competition (Holt [19]). In the non-spatial case where $d_{1}=d_{2}=d_{c}=0$, species with the higher value of $r_{k}{ }^{i} / c_{k}$ excludes the other resource species, if we can ignore density dependences in resource populations $\left(a_{k}{ }^{i}=0\right)$. This means that the resource (prey) species that wins is the one that maintains the highest density of shared consumer (predator), and this criterion was called the $P^{*}$ rule for apparent competition (Holt et al. [23]). In the following, we assume $d_{c}=0$ and mainly consider a question whether a locally inferior resource species (without loss of generality, we can assume that it is the second species) which becomes extinct in both patches can regionally survive or not when the dispersal rates satisfy some appropriate conditions.

The system has a steady state solution $E_{0}=\left(R_{1}{ }^{{ }^{*}}, R_{2}{ }^{1 *}, C^{1^{*}}, R_{1}{ }^{{ }^{*}}, R_{2}{ }^{2^{*}}, C^{2^{*}}\right)$, satisfying $R_{2}{ }^{1^{*}}=R_{2}{ }^{2^{*}}=0, R_{1}{ }^{i^{*}}=m^{i} /\left(b_{1} c_{1}\right), C^{i^{*}}=\left\{r_{1}{ }^{i}-a_{1}{ }^{i} m^{i} /\left(b_{1} c_{1}\right)-d_{1}(1-\right.$ $\left.\left.m^{j} / m^{i}\right)\right\} / c_{1}$ where $i, j=1,2$ and $j \neq i$. When $d_{1}=d_{2}=0$, we can easily prove that the steady state is globally stable if $C^{i *}>r_{2}{ }^{i} / c_{2}$, by constructing a Lyapunov function similar to the one used later for proof of global stability of a steady state when $d_{1} \neq 0$.

When $d_{1}>0$ and $d_{2}>0$, the characteristic equation at $E_{0}$ can be factorized into two-dimensional and four-dimensional polynomials. The latter has eigenvalues with negative real parts as long as $C^{i^{*}}>0$ is satisfied. The condition that the former has an eigenvalue with positive real part is equivalent to the one that the second resource can invade into the steady state. The steady state is stable if and only if

$$
\left(r_{2}{ }^{1}-c_{2} C^{1^{*}}\right)+\left(r_{2}^{2}-c_{2} C^{2^{*}}\right)-2 d_{2}<0
$$

and

$$
\left(r_{2}{ }^{1}-c_{2} C^{1^{*}}\right)\left(r_{2}{ }^{2}-c_{2} C^{2^{*}}\right)-\left\{\left(r_{2}{ }^{1}-c_{2} C^{1^{*}}\right)+\left(r_{2}{ }^{2}-c_{2} C^{2^{*}}\right)\right\} d_{2}>0 .
$$

Therefore, it is stable if $C^{i^{*}}>r_{2}{ }^{i} / c_{2}$ for $i=1,2$. However, since one of $C^{i^{*}}$ is a decreasing function of $d_{1}$ and the other is an increasing function unless $m^{1}=m^{2}$, one of $r_{2}{ }^{i}-c_{2} C^{i *}$ becomes positive and the other remains negative for sufficiently high values of $d_{1}$. Therefore, at least for $d_{2}=0$, the second condition becomes violated and the steady state loses stability if $d_{1}$ becomes sufficiently large. We can also find that increasing $d_{2}$ stabilizes the steady state if $d_{1}$ remains constant. Hereafter, we assume that $m^{1}>m^{2}$ without loss of generality. Since the mortality of consumer is higher in the first patch, apparent competition becomes weaker in this patch and coexistence of the two resource species is more readily realized. We also assume $d_{2}=0$ for our bifurcation analysis. 
At the point $C^{1^{*}}=r_{2}{ }^{1} / c_{2}$, a new steady state $E_{1}=\left(\widetilde{R}_{1}{ }^{1}, \widetilde{R}_{2}{ }^{1}, \widetilde{C}^{1}, \widetilde{R}_{1}{ }^{2}, \widetilde{R}_{2}{ }^{2}, \widetilde{C}^{2}\right)$, satisfying $\widetilde{R}_{2}{ }^{1}>0$ and $\widetilde{R}_{2}{ }^{2}=0$ bifurcates. Setting $\widetilde{R}_{1}{ }^{1}=z$, we can write the solution as follows;

$$
\begin{aligned}
& \widetilde{R}_{1}^{1}=z \\
& \widetilde{R}_{2}{ }^{1}=\frac{b_{1} c_{1}}{b_{2} c_{2}}\left(\frac{m^{1}}{b_{1} c_{1}}-z\right), \\
& \widetilde{C}^{1}=\frac{1}{c_{2}}\left\{r_{2}{ }^{1}-a_{2}{ }^{1} \frac{b_{1} c_{1}}{b_{2} c_{2}}\left(\frac{m^{1}}{b_{1} c_{1}}-z\right)\right\}, \\
& \widetilde{R}_{1}^{2}=\frac{m^{2}}{b_{1} c_{1}} \\
& \widetilde{R}_{2}^{2}=0 \\
& \widetilde{C}^{2}=\frac{1}{c_{1}}\left\{\frac{b_{1} c_{1}}{m^{2}}\left(z-\frac{m^{2}}{b_{1} c_{1}}\right) d_{1}+\left(r_{1}^{2}-a_{1}{ }^{2} \frac{m^{2}}{b_{1} c_{1}}\right)\right\} .
\end{aligned}
$$

$z$ is a unique positive solution of

$$
A z^{2}+\left(B_{1}+B_{2}\right) z+C=0
$$

where

$$
\begin{aligned}
& A=a_{1}{ }^{1} b_{2}\left(c_{2}\right)^{2}+a_{2}{ }^{1} b_{1}\left(c_{1}\right)^{2}, \\
& B_{1}=-\frac{m^{1}}{b_{1} c_{1}} A+d_{1} b_{2}\left(c_{2}\right)^{2} \frac{m^{2}}{m^{1}} \\
& B_{2}=b_{2} c_{1} c_{2}\left(r_{2}{ }^{1}-c_{2} C^{1^{*}}\right), \\
& C=-d_{1} \frac{m^{2} b_{2}\left(c_{2}\right)^{2}}{b_{1} c_{1}}
\end{aligned}
$$

We define a function of $z, h(z)=A z^{2}+\left(B_{1}+B_{2}\right) z+C$. Since

$$
A z^{2}+B_{1} z+C=0, \quad \text { if } \quad z=\frac{m^{1}}{b_{1} c_{1}},
$$

$h\left(m^{1} /\left(b_{1} c_{1}\right)\right)=B_{2} m^{1} /\left(b_{1} c_{1}\right) \geq 0$, and

$$
h\left(\frac{m^{2}}{b_{1} c_{1}}\right)=\frac{m^{2}}{b_{1} c_{1}}\left(\frac{m^{2}}{b_{1} c_{1}}-\frac{m^{1}}{b_{1} c_{1}}\right) A+b_{2} c_{1} c_{2}\left[r_{2}{ }^{1}-\frac{c_{2}}{c_{1}}\left(r_{1}{ }^{1}-a_{1}{ }^{1} \frac{m^{1}}{b_{1} c_{1}}\right)\right]<0
$$

is satisfied if $E_{0}$ is stable when $d_{1}=d_{2}=0$.

Therefore, the equation $h(z)=0$ has a unique positive solution between $m^{2} /\left(b_{1} c_{1}\right)$ and $m^{1} /\left(b_{1} c_{1}\right)$ and $\widetilde{R}_{2}^{1} \geq 0 . \quad \widetilde{C}^{2}$ is positive if it is so when $d_{1}=0$. If $d_{1}$ is not too large or the condition $m^{1}-m^{2}<b_{2} c_{2} r_{2}{ }^{1} / a_{2}{ }^{1}$ is satisfied, then $\widetilde{C}^{1}$ is also positive. 
Global stability of $E_{1}$ can be proved by constructing a Lyapunov function as follows. Define

$$
\varphi(x)=x-\tilde{x}-\tilde{x} \log \frac{x}{\tilde{x}},
$$

and

$$
V_{E_{1}}=\widetilde{R}_{1}^{1}\left\{b_{1} \varphi\left(R_{1}{ }^{1}\right)+b_{2} \varphi\left(R_{2}{ }^{1}\right)+\varphi\left(C^{1}\right)\right\}+\widetilde{R}_{1}{ }^{2}\left\{b_{1} \varphi\left(R_{1}{ }^{2}\right)+b_{2} R_{2}{ }^{2}+\varphi\left(C^{2}\right)\right\} .
$$

$V_{E_{1}}$ is positive definite and vanishes only at the steady state $E_{1}$.

Then,

$$
\begin{aligned}
\frac{d V_{E_{1}}}{d t}= & -\widetilde{R}_{1}{ }^{1}\left\{b_{1} a_{1}{ }^{1}\left(R_{1}{ }^{1}-\widetilde{R}_{1}{ }^{1}\right)^{2}+b_{2} a_{2}{ }^{1}\left(R_{2}{ }^{1}-\widetilde{R}_{2}{ }^{1}\right)^{2}\right\} \\
& -\widetilde{R}_{1}{ }^{2}\left\{b_{1} a_{1}{ }^{2}\left(R_{1}{ }^{2}-\widetilde{R}_{1}{ }^{2}\right)^{2}+b_{2} a_{2}{ }^{2}\left(R_{2}{ }^{2}\right)^{2}\right\} \\
& +\widetilde{R}_{2}{ }^{1} b_{2}\left(r_{2}{ }^{2}-c_{2} \widetilde{C}^{2}\right) R_{2}{ }^{2} \\
& -d_{1} b_{1} \frac{\left(\widetilde{R}_{1}{ }^{1} \widetilde{R}_{1}{ }^{2}\right)^{2}}{R_{1}{ }^{1} R_{1}{ }^{2}}\left(\frac{R_{1}{ }^{1}}{\widetilde{R}_{1}{ }^{1}}-\frac{R_{1}{ }^{2}}{\widetilde{R}_{1}{ }^{2}}\right)^{2} .
\end{aligned}
$$

For $d_{1}>0, \widetilde{C}^{2}$ is an increasing function of $z$ and $r_{2}{ }^{2}-c_{2} \widetilde{C}^{2}$ is a decreasing function of $z$. However, even if $z=m^{2} /\left(b_{1} c_{1}\right)$,

$$
r_{2}{ }^{2}-c_{2} \widetilde{C}^{2}=r_{2}{ }^{2}-\frac{c_{2}}{c_{1}}\left(r_{1}{ }^{2}-a_{1}{ }^{2} \frac{m^{2}}{b_{1} c_{1}}\right)<0
$$

if it is negative for $d_{1}=0$. Therefore, equation (20) is negative definite and the invariant set in which it vanishes is the steady state $E_{1}$. This completes the proof of global stability of $E_{1}$.

We have found that, as $d_{1}$ increases, the competitive pressure on the inferior resource in the first patch lessens and $\widetilde{R}_{2}{ }^{1}$ becomes positive, since $\widetilde{R}_{1}{ }^{1}$ is smaller than $m^{1} /\left(b_{1} c_{1}\right)$. If $d_{2}$ is positive, the inferior resource flows from the first patch to the second and we can expect that a positive steady state bifurcates as the condition (4) is violated. We numerically verify this prediction. In Fig. 3, we assumed the following values of parameters; $r_{1}{ }^{1}=r_{2}{ }^{1}=r_{1}{ }^{2}=r_{2}{ }^{2}=1, a_{1}{ }^{1}=a_{2}{ }^{1}=a_{1}{ }^{2}=$ $a_{2}^{2}=0.1, c_{1}=1, c_{2}=2, b_{1}=1, b_{2}=0.5, m^{1}=2, m^{2}=1, d_{c}=0$. A dot at a grid point in a space of $d_{1}-d_{2}$ denotes that a positive steady state exists for the combination of dispersal rates $d_{1}$ and $d_{2}$. The fat curve on the left boundary of the coexistence region is a set of bifurcation points. Across the boundary a positive steady state bifurcates from $E_{0}$. However, across the upper boundary, $\widetilde{C}^{1}$ becomes zero before $\widetilde{R}_{2}{ }^{1}$ and $\widetilde{R}_{2}{ }^{2}$ vanish. Therefore, it is much more difficult to study a bifurcation phenomenon across the upper boundary, since we must solve a steary state satisfying $\widetilde{C}^{1}=0$ for $d_{1}>0$ and $d_{2}>0$. Thus we skip it here. Fig. 3 clearly shows that the higher dispersal rate of the superior competitor and 


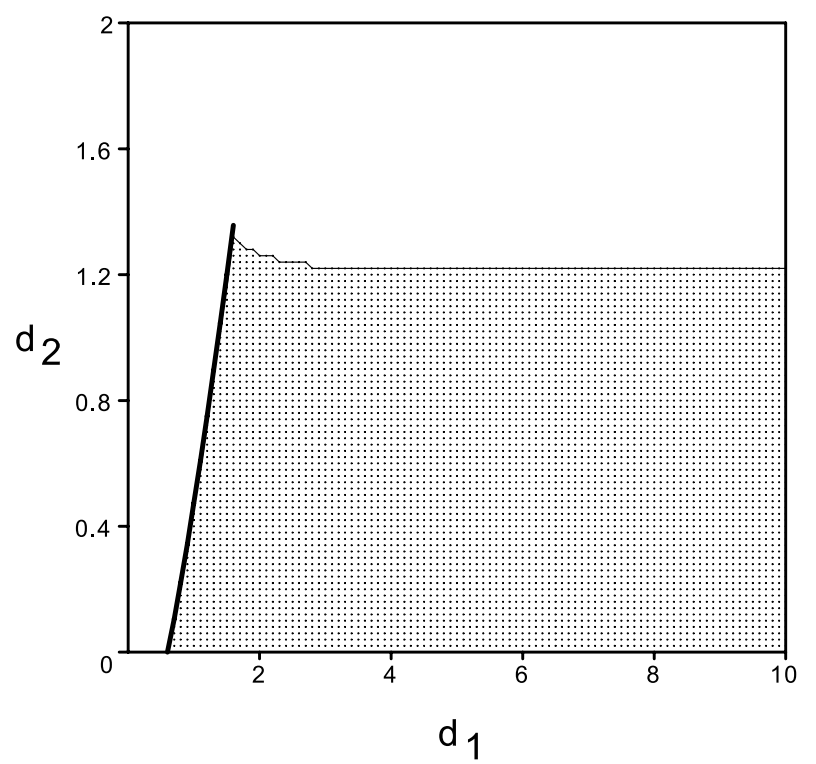

Fig. 3. Conditions for dispersal-mediated coexistence in a parameter space $d_{1}-d_{2}$. A dot at a grid point denotes that a positive steady state exists for the combination of dispersal rates $d_{1}$ and $d_{2}$. The fat curve on the left boundary of the coexistence region is a set of bifurcation points. Across the boundary a positive stable steady state bifurcates from $E_{0}$. However, across the upper boundary, $\widetilde{C}^{1}$ becomes zero before $\widetilde{R}_{2}{ }^{1}$ and $\widetilde{R}_{2}{ }^{2}$ vanish. Therefore, above the coexistence region, there exists a stable steady state satisfying either (A) $R_{2}{ }^{1}>0, R_{2}{ }^{2}>0$, and $C^{1}=0$ or (B) $R_{2}{ }^{1}=R_{2}{ }^{2}=C^{1}=0$. Values of parameters are as follows; $r_{1}{ }^{1}=r_{2}{ }^{1}=r_{1}{ }^{2}=r_{2}{ }^{2}=1, a_{1}{ }^{1}=a_{2}{ }^{1}=a_{1}{ }^{2}=a_{2}{ }^{2}=0.1, c_{1}=1, c_{2}=2, b_{1}=1$, $b_{2}=0.5, m^{1}=2, m^{2}=1, d_{c}=0$.

the lower dispersal rate of the inferior competitor promote coexistence of apparent competitors just as well in the case of exploitative competition.

Hitherto, we only considered the case where the competitive rankings are the same in both patches. Here, we consider the case in which each species has a source and a sink. Thus, either of $r_{k}{ }^{i}-c_{k} C^{i *}(i=1,2)$ is negative and the other is positive. If the second species can move between the source and sink patches, the condition (4) is violated at least for small $d_{2}$, and $E_{0}$ becomes unstable. However, if $\left(r_{2}{ }^{1}-c_{2} C^{1^{*}}\right)+\left(r_{2}{ }^{2}-c_{2} C^{2^{*}}\right)<0$ (the second species is an overall inferior competitor when averaged across the metacommunity), both (3) and (4) are satisfied for large $d_{2}$, and $E_{0}$ becomes stable. Therefore, there is a critical maximum dispersal rate over which the overall inferior competitor goes to extinction when there is a true source-sink structure (Amarasekare and Nisbet [5]).

When the first patch with the higher consumer mortality is a sink $\left(r_{2}{ }^{1}-\right.$ $\left.c_{2} C^{1^{*}}<0\right)$ and the second patch is a source $\left(r_{2}{ }^{2}-c_{2} C^{2 *}>0\right)$ for the second species, the source will turn to a sink $\left(r_{2}{ }^{2}-c_{2} C^{2 *}<0\right)$ and the sink to a source $\left(r_{2}{ }^{1}-c_{2} C^{1^{*}}>0\right)$ as $d_{1}$ increases, since the consumer abundances $C^{1^{*}}$ and $C^{2^{*}}$ 
are a decreasing and an increasing function of $d_{1}$ respectively (recall that $C^{i^{*}}=$ $\left\{r_{1}{ }^{i}-a_{1}{ }^{i} m^{i} /\left(b_{1} c_{1}\right)-d_{1}\left(1-m^{j} / m^{i}\right)\right\} / c_{1}$ for $i, j=1,2$ and $j \neq i$, and that it does not depend on $d_{2}$ ). However, it should be noted that for some parameter values (for example, if we change only the value of $r_{2}{ }^{2}$ to 2.5 in the example of Fig. 3), both patches become sinks of the second species $\left(r_{2}{ }^{i}-c_{2} C^{i^{*}}<0\right.$ for $\left.i=1,2\right)$ and $E_{0}$ turns stable for intermediate values of $d_{1}$ when $d_{2}=0$. As $d_{1}$ becomes larger, the first patch turns to a source for the second species $\left(r_{2}{ }^{1}-c_{2} C^{1^{*}}>0\right)$ and the species can persist if $d_{2}$ is small. However, if $d_{2}$ is too large, the second species becomes extinct since $\left(r_{2}{ }^{1}-c_{2} C^{1^{*}}\right)+\left(r_{2}{ }^{2}-c_{2} C^{2 *}\right)<0$ and equation (4) is satisfied for large $d_{2}$. Thus, intermediate dispersal rates of competitors may be harmful for a species in a true source-sink environment (Amarasekare and Nisbet [5]).

\section{Discussion}

We studied two metacommunity models of indirect competition in a habitat of two different patches. The first is a model of two consumers (predators) sharing a common resource (prey) and the second is a model of two resources (prey) sharing a common consumer (predator). We have shown in both models that two indirect competitors can coexist in a habitat of patches connected through dispersal of individuals, even if their competitive rankings are spatially homogeneous and the inferior species becomes driven to extinction in any patches in isolation.

In a natural habitat, some demographic parameters, for example, resource growth rates, carrying capacities or consumer mortalities, usually differ among patches. Then, populations become more abundant in more favorable patches and, if the patches are connected through dispersal, the populations flow from the more favorable patches to the less favorable ones. Thus, any spatial heterogeneity creates asymmetric population distributions in the habitat. However, this does not necessarily imply emergence of the source-sink dynamics since some species might go to extinction, if they are competitively inferior or inefficient in resource use in any patches throughout the habitat. When the competitive rankings of indirect competitors do not vary over space, any patch becomes a source for the winner and a sink for the loser. However, because of slight spatial heterogeneity that is insufficient to reverse the competitive rankings, there appear population flows even in this case from the relatively favorable patches to the relatively unfavorable ones. Therefore, some of the source patches accept immigrants and take parts of "pseudo-sinks" in the sense of Watkinson and Sutherland [69].

If the competitive rankings are spatially invariant and any patches are sinks for inferior competitors, their own dispersal alone cannot save them from extinction. However, there are at least two spatial mechanisms to create sourse-sink spatial structures in populations of inferior competitors and save them from regional extinction. (1) Source-sink (pseudosink) spatial structure of the superior competitors induces emigration of their populations from the more favorable patches and release competitive pressures on the inferior in these patches. (2) Resource flows into patches with the lower consumer mortalities and lower resource abundance enhance 
(or subsidize) growth of the inferior exploitative competitors and save the populations from extinction. Once the populations in the more productive patches in the first case or in the patches with the lower consumer mortalities in the second case become established, emigrants from the patches can save populations of the inferior competitors in the other patches. Thus, for the inferior indirect competitors, there emerges a spatial structure of a "relative source" and a "relative sink" (Pacala and Roughgarden [55]) or an "apparent source" and an "apparent sink" (Namba and Hashimoto [51]) in a habitat of sole sinks. Since the relative or apparent source exporting individuals of the inferior species is a sink in isolation, it might be possible to call it a 'pseudo-source.'

Metacommunity dynamics depends on the degree of spatial heterogeneity (Mouquet et al., [46]) and the magnitude of dispersal rates. We found that spatial coexistence of indirect competitors in metacommunities does not require so high degree of heterogeneity to reverse the competitive rankings within the habitat. When local abiotic conditions or biotic interactions in a patch prevent a species from maintaining a population in isolation, dispersal of competitors or resources owing to their source-sink spatial structures may favor the inferior species and save it from extinction. This means that not only the dispersal rate of the species itself but also the dispersal rates of interacting species determine the spatial structure of a species in a metacommunity.

The competition-colonization trade-off and source-sink dynamics are the two major hypothesis to explain coexistence of competitors in a metacommunity. Our mechanism of coexistence belongs to the source-sink dynamics in a broader sense. However, it does not rely on high degree of spatial heterogeneity to make competitive rankings spatially variable. Thus, to infer which mechanism, ours or the sourcesink dynamics in a narrower sense, is relevant to explain empirically found patterns, it is necessary to measure the degree of spatial heterogeneity in the metacommunity. However, it is not easy to assess competitive rankings in isolated patches across the habitat. It may be necessary not only to measure demographic parameters and interaction strengths but also to enclose habitat patches for isolating them. Since the habitat is nearly homogeneous in our model, it is necessary to determine which of the two mechanisms, ours or the competition-colonization trade-off, is appropriate to explain empirically observed spatial coexistence. If the dispersal rate of locally inferior species is found to be low, then the competition-colonization trade-off is irrelevant. However, it may also be a tough task to estimate dispersal rates and new techniques will be necessary (Schneeberger and Jansen [58]).

In the metacommunity model of apparent competition, we neglected consumer dispersal. Thus, differences in consumer mortalities between patches were necessary for dispersal to destabilize the steady state at which the inferior species is extinct. However, our preliminary numerical analysis showed that dispersal-mediated coexistence is possible even if the consumer mortality is identical in the two patches when the consumer can move between the patches. Thus it may be an interesting future question to reveal the effect of consumer dispersal on spatial coexistence of apparent competitors. To date, most metacommunity models assume spatially 
discrete habitats. A few spatially continuous models are either the spatial point processes (Bolker and Pacala [7], Murrell and Law [49]) or the lottery models (Snyder and Chesson [61]), with dispersal and competition kernels. The reason may lie in the difficulty to analyze continuous models with spatial heterogeneity in parameter values. To study another kind of spatially continuous models, such as the reaction-diffusion models (Shigesada and Kawasaki [59], Okubo and Levin [53]), will be an important future challenge.

Acknowledgement. I thank Professors Masayasu Mimura and Yasuhiro Takeuchi for inviting me to this special issue of JJIAM. This work was partially supported by Grants-in-Aid for Scientific Research (C) 13640634 and (A) 13304006 from Japan Society for the Promotion of Science and by the Osaka Prefectural Government through the Special Joint Research Project for Environmental Sciences.

\section{References}

[1] P. Abrams and W.G. Wilson, Coexistence of competitors in metacommunities due to spatial variation in resource growth rates; does $\mathrm{R}^{*}$ predict the outcome of competition? Ecol. Lett., 7, (2004) 929-940.

[ 2 ] F.R. Adler and J. Mosquera, Is space necessary? Interference competition and limits to biodiversity, Ecology, 81, (2000) 3226-3232.

[ 3 ] P. Amarasekare, Competitive coexistence in spatially structured environments: a synthesis. Ecol. Lett., 6, (2003) 1109-1122.

[ 4 ] P. Amarasekare, M.F. Hoopes, N. Mouquet and M. Holyoak, Mechanisms of coexistence in competitive metacommunities. Am. Nat., 164, (2004) 310-326.

[ 5 ] P. Amarasekare and R.M. Nisbet, Spatial heterogeneity, source-sink dynamics, and the local coexistence of competing species. Am. Nat., 158, (2001) 572-584.

[ 6 ] R.A. Armstrong and R. McGehee, Competitive exclusion. Am. Nat., 115, (1980) 151-170.

[ 7 ] B.M. Bolker and S.W. Pacala, Spatial moment equations for plant communities: understanding spatial strategies and the advantages of short dispersal. Am. Nat., 153, (1999) 575-602.

[ 8 ] J.H. Brown and A. Kodric-Brown, Turnover rates in insular biogeography: effect of immigration on extinction. Ecology, 58, (1977) 445-449.

[ 9 ] V. Calcagno, N. Mouquet, P. Jarne and P. David, Coexistence in a metacommunity: the competition-colonization trade-off is not dead. Ecol. Lett., 9, (2006) 897-907.

[10] R.S. Cantrell and C. Cosner, On the effects of spatial heterogeneity on the persistence of interacting species. J. Math. Biol., 37, (1998) 103-145.

[11] J.M. Chase, P. Amarasekare, K. Cottenie, A. Gonzalez, R.D. Holt, M. Holyoak, M.F. Hoopes, M.A. Leibold, M. Loreau, N. Mouquet, J.B. Shurin and D. Tilman, Competing theories for competitive metacommunities. Metacommunities: Spatial Dynamics and Ecological Communities, (M. Holyoak, M.A. Leibold and R.D. Holt eds.), Chicago University Press, Chicago, 2005, 335-354.

[12] P. Chesson, Coexistence of competitors in spatially and temporally varying environments: a look at the combined effects of different sorts of variability. Theor. Popul. Biol., 28, (1985) 263-287.

[13] P. Chesson, General theory of competitive coexistence in spatially-varying environments. Theor. Popul. Biol., 58, (2000) 211-237.

[14] P. Chesson, Mechanisms of maintenance of species diversity. Annu. Rev. Ecol. Syst., 31, (2000) 343-366.

[15] U. Dieckmann, R. Law and J.A.J. Metz (eds.), The Geometry of Ecological Interactions: Simplifying Spatial Complexity, Cambridge University Press, Cambridge, 2000. 
[16] I.A. Hanski and M.E. Gilpin, Metapopulation dynamics: brief history and conceptual domain. Biol. J. Linn. Soc., 42, (1991) 3-16.

[17] I.A. Hanski and M.E. Gilpin (eds.), Metapopulation Biology: Ecology, Genetics, and Evolution. Academic Press, San Diego, 1997

[18] A. Hastings, Disturbance, coexistence, history, and competition for space. Theor. Popul. Biol., 18, (1980) 363-373.

[19] R.D. Holt, Predation, apparent competition, and the structure of prey communities. Theor. Popul. Biol., 12, (1977) 197-229.

[20] R.D. Holt, Spatial heterogeneity, indirect interactions, and coexistence of prey species. Am. Nat., 124, (1984) 377-406.

[21] R.D. Holt, Population dynamics in two-patch environments: some anomalous consequences of optimal habitat selection. Theor. Popul. Biol., 28, (1985) 181-208.

[22] R.D. Holt, Ecology at the mesoscale: the influence of regional processes on local communities. Species Diversity in Ecological Communities: Historical and Geographical Perspectives, (R.E. Ricklefs and D. Schulter eds.), University of Chicago Press, Chicago, 1993, 77-88.

[23] R.D. Holt, J. Grover and D. Tilman, Simple rules for interspecific dominance in systems with exploitative and apparent competition. Am. Nat., 144, (1994) 741-771.

[24] M. Holyoak and S.P. Lawler, The role of dispersal in predator-prey metapopulation dynamics. J. Anim. Ecol., 65, (1996) 640-652.

[25] M. Holyoak and S.P. Lawler, Persistence of an extinction-prone predator-prey interaction through metapopulation dynamics. Ecology, 77, (1996) 1867-1879.

[26] M. Holyoak, M.A. Leibold and R.D. Holt editors, Metacommunities: Spatial Dynamics and Ecological Communities. Chicago University Press, Chicago, 2005.

[27] M. Holyoak, M.A. Leibold, N. Mouquet, R.D. Holt, M.F. Hoopes, Metacommunities. A framework for large-scale community ecology. Metacommunities: Spatial Dynamics and Ecological Communities, (M. Holyoak, M.A. Leibold and R.D. Holt, eds.), Chicago University Press, Chicago, 2005, 35-67.

[28] M.F. Hoopes, R.D. Holt and M. Holyoak, The effects of spatial processes on two species interactions. Metacommunities: Spatial Dynamics and Ecological Communities, (M. Holyoak, M.A. Leibold and R.D. Holt eds.), Chicago University Press, Chicago, 2005, 35-67.

[29] H.S. Horn and R.H. MacArthur, Competition among fugitive species in a harlequin environment. Ecology, 53, (1972) 749-752.

[30] S.B. Hsu, S.P. Hubbell and P. Waltman, Competing predators. SIAM J. Appl. Math., 35, (1978) 617-625.

[31] S.B. Hsu, S.P. Hubbell and P. Waltman, A contribution to the theory of competing predators. Ecol. Monog., 48, (1978) 337-349.

[32] Y. Iwasa and J. Roughgarden, Interspecific competition among metapopulations with spacelimited subpopulations. Theor. Popul. Biol., 30, (1986) 194-214.

[33] K. Kishimoto, Coexistence of any number of species in the Lotka-Volterra competitive system over two patches. Theor. Popul. Biol., 38, (1990) 149-158.

[34] J.M. Kneitel and J.M. Chase, Trade-offs in community ecology: linking spatial scales and species coexistence. Ecol. Lett., 7, (2004) 69-80.

[35] A.L. Koch, Competitive coexistence of two predators utilizing the same prey under constant environmental conditions. J. Theor. Biol., 44, (1974) 387-395.

[36] V.A. Kostitzin, Biologie Mathématique. Collection Armand Colin: Paris, Translated in English by T.H. Savory in 1939. Mathematical Biology. Harrap and Co., London, 1937.

[37] M.A. Leibold, M. Holyoak, N. Mouquet, P. Amarasekare, J.M. Chase, M.F. Hoopes, R.D. Holt, J.B. Shurin, R. Law, D. Tilman, M. Loreau and A. Gonzalez, The metacommunity concept: a framework for multi-scale community ecology. Ecol. Lett., 7, (2004) 601-613.

[38] J. Leon and D. Tumpson, Competition between two species for two complementary or substitutable resources. J. Theor. Biol., 50, (1975) 185-201.

[39] S.A. Levin, Dispersion and population interactions. Am. Nat., 108, (1974) 207-228.

[40] R. Levins, Some demographic and genetic consequences of environmental heterogeneity for biological control. Bull. Entomol. Soc. Am., 15, (1969) 237-240.

[41] R. Levins, Extinction. Some Mathematical Problems in Biology, (M. Gerstenhaber, ed.), American Mathematical Society: Providence RI. 1970, 75-107. 
[42] R. Levins and D. Culver, Regional coexistence of species and competition between rare species. Proc. Natl. Acad. Sci. USA, 68, (1971) 1246-1248.

[43] N. Mouquet, M.F. Hoopes and P. Amarasekare, The world is patchy and heterogeneous! Trade-off and source-sink dynamics in competitive metacommunities. Metacommunities: Spatial Dynamics and Ecological Communities, (M. Holyoak, M.A. Leibold and R.D. Holt, eds.), Chicago University Press, Chicago, 2005, 237-262.

[44] N. Mouquet and M. Loreau, Coexistence in metacommunities: the regional similarity hypothesis. Am. Nat., 159, (2002) 420-426.

[45] N. Mouquet and M. Loreau, Community patterns in source-sink metacommunities. Am. Nat., 162, (2003) 544-557.

[46] N. Mouquet, T.E. Miller, T. Daufresne and J.M. Kneitel, Consequences of varying regional heterogeneity in source-sink metacommunities, Oikos, 113, (2006) 481-488.

[47] S. Muko and Y. Iwasa, Species coexistence by permanent spatial heterogeneity in a lottery model. Theor. Popul. Biol., 57, (2000) 273-284.

[48] S. Muko and Y. Iwasa, Incomplete mixing promotes species coexistence in a lottery model with permanent spatial heterogeneity. Theor. Popul. Biol., 64, (2003) 359-368.

[49] D.J. Murrell and R. Law, Heteromyopia and the spatial coexistence of similar competitors. Ecol. Lett., 6, (2003) 48-59.

[50] T. Namba, Emigration of a population and stability of a prey-predator system. Bull. Assoc. Nat. Sci. Senshu Univ., 11, (1993) 9-20.

[51] T. Namba and C. Hashimoto, Dispersal-mediated coexistence of competing predators. Theor. Popul. Biol., 66, (2004) 53-70.

[52] T. Namba, A. Umemoto and E. Minami, The Effects of Habitat Fragmentation on Persistence of Source-Sink Metapopulations in Systems with Predators and Prey or Apparent Competitors. Theor. Popul. Biol., 56, (1999) 123-137.

[53] A. Okubo and S.A. Levin, Diffusion and Ecological Problems: Modern Perspectives (Interdisciplinary Applied Mathematics). Springer-Verlag, New York, 2001.

[54] S.W. Pacala and M. Rees, Models suggesting field experiments to test two hypotheses explaining successional diversity. Am. Nat., 152, (1998) 729-737.

[55] S.W. Pacala, and J. Roughgarden. Spatial heterogeneity and interspecific competition. Theor. Popul. Biol., 21, (1982) 92-113.

[56] H.R. Pulliam, Sources, sinks, and population regulation. Am. Nat., 132, (1988) 652-661.

[57] H.R. Pulliam and B.J. Danielson, Sources, sinks, and habitat selection: a landscape perspective on population dynamics. Am. Nat., 137, (1991) S50-S66.

[58] A. Schneeberger and V.A.A. Jansen, The estimation of dispersal rates using the covariance of local populations. Ecol. Modell., 196, (2006) 434-446.

[59] N. Shigesada and K. Kawasaki, Biological Invasions: Theory and Practice (Oxford Series in Ecology and Evolution). Oxford University Press, Oxford, New York, 1997.

[60] A. Shmida and R.H. Whittaker, Pattern and biological microsite effects in two shrub communities, Southern California. Ecology, 62, (1981) 234-251.

[61] R. Snyder and P. Chesson, Local dispersal can facilitate coexistence in the presence of permanent spatial heterogeneity. Ecol. Lett., 6, (2003) 301-309.

[62] F.M. Stewart and B.R. Levin, Partitioning of resources and the outcome of interspecific competition: a model and some general considerations. Am. Nat., 107, (1973) 171-198.

[63] Y. Takeuchi, Diffusion-mediated persistence in two-species competition Lotka-Volterra model. Math. Biosci., 95, (1989) 65-83.

[64] D. Tilman, Resources: a graphical-mechanistic approach to competition and predation. Am. Nat., 116, (1980) 362-393.

[65] D. Tilman, Resource Competition and Community Structure. Princeton University Press, Princeton, 1982.

[66] D. Tilman, Competition and biodiversity in spatially structured habitats. Ecology, 75, (1994) 2-16.

[67] D. Tilman and P. Kareiva, eds., Spatial Ecology: The Role of Space in Population Dynamics and Interspecific Interactions. Princeton University Press, Princeton, 1997.

[68] D. Tilman, R.M. May, C.L. Lehman and M.A. Nowak, Habitat destruction and the extinction debt. Nature, 371, (1994) 65-66. 
[69] A.R. Watkinson and W.J. Sutherland, Sources, sinks and pseudo-sinks. J. Anim. Ecol., 64, (1995) 126-130.

[70] D.S. Wilson, Complex interactions in metacommunities with implications for biodiversity and higher levels of selection. Ecology, 73, (1992) 1984-2000.

[71] D.W. Yu and H.B. Wilson, The competition-colonization trade-off is dead; long live the competition-colonization trade-off. Am. Nat., 158, (2001) 49-63. 
